\title{
QUALITY IMPROVEMENT Exception reporting: an effective way to increase compliance leading to improvements in working conditions for junior doctors
}

\author{
Authors: Dhanuja Senn, ${ }^{\mathrm{A}}$ Ruwani Mawella ${ }^{\mathrm{A}}$ and Myooran Ganeshananthan ${ }^{\mathrm{B}}$
}

\section{Background}

In 2016, the new junior doctors contract introduced exception reporting (ER) to monitor extra hours worked, report patient safety, rostering and training concerns. Unfortunately, from discussions with foundation doctors, there seems to be a reluctance to engage with ER.

\section{Objectives}

This quality improvement project aims to identify reasons for reluctance among foundation doctors to engage with ER and implement changes to address these barriers with a view to improve rates of ER.

Methods

Questionnaires regarding ER were distributed to all foundation doctors at a district general hospital. Following this, a leaflet was created and disseminated to all doctors providing guidance on ER and addressing several issues highlighted by the questionnaire. Foundation doctors were re-surveyed to assess for improvement.

Results

We found a significant improvement $(p<0.05)$ in proportion of overtime exception reports filled from $20.3 \%$ to $33.9 \%$. The most common barriers identified were apprehension of meeting with the consultant to discuss reports, insufficient senior encouragement and fear of appearing inefficient.

\section{Conclusions}

An increased awareness and understanding of ER through the leaflet achieved an improvement in rates of ER. The insight gained from this quality improvement report can be applied across other trusts to improve compliance with the ER system nationwide, in order to safeguard staff wellbeing and thereby improve patient safety.

KEYWORDS: patient safety, exception reporting, junior doctors, safe working

DOI: $10.7861 /$ fhj.2020-0023

Authors: Afoundation doctor, Royal Surrey County Hospital, Guildford, UK; ${ }^{B}$ consultant, Royal Surrey County Hospital, Guildford, UK

\section{Introduction}

The European Working Directive (2003/88/EC) mentions that safe working hours should average 48 hours a week, with adequate breaks throughout shifts. This aims to reduce burnout and stress, and improve the health and safety of the workforce. The increasingly busy nature of the hospital means that junior doctors regularly work above and beyond their contractual requirements. ${ }^{1}$ In 2016, exception reporting (ER) was introduced in the new junior doctors' contract with the aim being to monitor extra hours worked, report patient safety, rostering and training concerns, and subsequently be reimbursed in the form of pay or time off. ${ }^{2}$ This system is endorsed by the General Medical Council (GMC) and British Medical Association (BMA) and replaced the old hours monitoring and banding system from the 2002 contract, whereby disputes on correct banding could only be resolved through a formal banding appeal. ${ }^{1,3}$ Dr Mairi Reid, BMA junior doctors committee deputy chair, states:

The exception reporting system is vital in ensuring doctors are encouraged and feel able to highlight times when their working hours and shift patterns are non-compliant and - ultimatelyunsafe.

Unfortunately, from discussions with foundation doctors, there seems to be a reluctance among trainees to engage with ER. This appears to be consistent among other trusts. ${ }^{4}$ Due to the novelty of the ER system, there is a scarcity of data identifying the reasons behind this. At the district general hospital where this study was executed, $76 \%$ of junior doctors had not submitted a report between August 2017 and June 2018. ${ }^{5}$ This may be a reflection on challenges relating to organisational culture, staff engagement or reporting processes. ${ }^{6}$

The wellbeing of doctors is vital because there is abundant evidence that workplace stress in healthcare organisations affects quality of care for patients as well as doctors' own health. ${ }^{7}$

Identifying and, in turn, addressing the challenges surrounding ER will allow chronically understaffed and overstretched departments to be identified, so that trust boards can address such issues. ${ }^{8}$ These improvements in working conditions and, therefore, junior doctor wellbeing will, in turn, lead to enhanced patient safety. ${ }^{9}$ 
This quality improvement project aimed to identify the reasons for the reluctance among foundation trainees to engage with ER. On the basis of the results, changes were implemented to address these barriers, with a view to improve rates of ER and the additional beneficial effect of improving patient safety.

\section{Methods}

Plan, do, study, act cycle 1

A mixed methods research design was used. The junior doctor forum, to which all trainees were invited, was used as a platform to discuss concerns about using ER as a means to highlight rota gaps or lack of access to educational opportunities. Thematic analysis of the results from the forum formed the basis of our questionnaire. Anonymised paper questionnaires (supplemental material S1) were distributed to all foundation trainees (a cohort of 62) working at the district general hospital to identify rates of ER for overtime worked over a 2-month period. Other questions included level of trainee satisfaction with the ER process and the perceived barriers to ER.

Thematic analysis of the results of the questionnaire allowed us to identify the most common perceived barriers. This was used to create a leaflet (Fig 1) to provide guidance on the ER

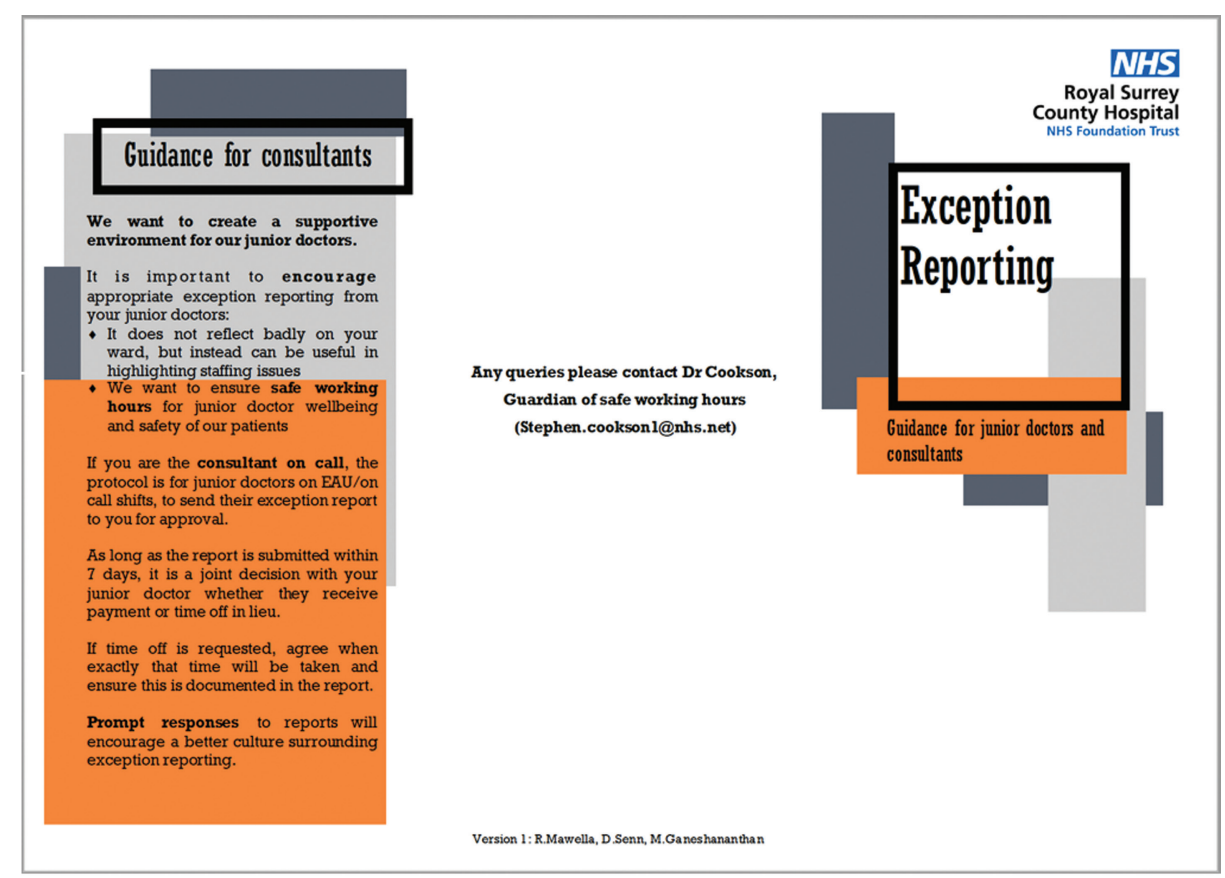

\begin{tabular}{|c|c|c|}
\hline & How to submit an ER & Guidance for junior doctors \\
\hline What is exception reporting? & 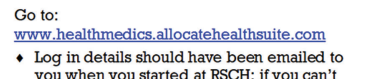 & $\begin{array}{l}\text { Exception reporting does not signify } \\
\text { inefficiency, butrather is is important way of } \\
\text { identifying high workload and lack of } \\
\text { stafffing. }\end{array}$ \\
\hline \multirow{6}{*}{$\begin{array}{l}\text { A process that has been put in place to } \\
\text { allow you to flag up when your work } \\
\text { has varied from your agreed work } \\
\text { schedule } \\
\text { You should exception report issues as } \\
\text { they arise including: } \\
\text { - Difierences in total hours worked } \\
\text { (iie. overtime) } \\
\text { R Rest treaks not taken (you should } \\
\text { have a } 30 \text { minute break for a shift } \\
>5 \text { hours and another } 30 \text { minute } \\
\text { break if its }>\text { hours } \\
\text { - Educational opportunities or } \\
\text { training missed } \\
\text { - Lack of support available }\end{array}$} & tr.doctorsxceptionteporting@nhs.net & $\begin{array}{l}\text { Any length of overtime is acceptable to } \\
\text { submit a report for (even } 15 \text { minutes). }\end{array}$ \\
\hline & following screen should appear: & $\begin{array}{l}\text { On an EAU/on-call shift, submit reports to } \\
\text { the consultant on call for that shift, }\end{array}$ \\
\hline & $=$ & $\begin{array}{l}\text { site at the time. (They have been made } \\
\text { aware of this) }\end{array}$ \\
\hline & - & $\begin{array}{l}\text { The consultant may wish to meet with you to } \\
\text { discuss the report. This is for supportive } \\
\text { purposes. }\end{array}$ \\
\hline & 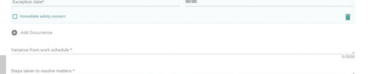 & $\begin{array}{l}\text { If there is a delay in approval of your reports, } \\
\text { the guardian of safe working hours (Dr } \\
\text { Cookson) will help to chase this up. }\end{array}$ \\
\hline & 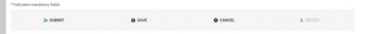 & If time off is requested, agree when exactly \\
\hline \multirow{2}{*}{$\begin{array}{l}\text { This allows for changes to be made } \\
\text { and for you to be paid or have time off } \\
\text { in lieu for any additional time you've } \\
\text { worked. }\end{array}$} & & $\begin{array}{l}\text { that time will be taken and ensure this is } \\
\text { documented in the report. }\end{array}$ \\
\hline & $\begin{array}{l}\text { For further guidance on how to complete the } \\
\text { report and ex amples go to to: } \\
\text { Gdrive/shared/Medicine/ExceptionReporting }\end{array}$ & \multirow{2}{*}{$\begin{array}{l}\text { After the consultant has responded to your } \\
\text { report, you must review it and agree/ } \\
\text { disagree with the outcome before it can be } \\
\text { sent to payroll. }\end{array}$} \\
\hline $\begin{array}{l}\text { There are no restrictions on what } \\
\text { should be reported or how many } \\
\text { reports you can submit. }\end{array}$ & $\begin{array}{l}\text { It is advised that you state your preference (ie. } \\
\text { time off/payment) in the free text area. }\end{array}$ & \\
\hline $\begin{array}{l}\text { Exception reporting is a contract } \\
\text { based requirement. Doctors are } \\
\text { expected to comply with a system that } \\
\text { monitors hours worked. }\end{array}$ & $\begin{array}{l}\text { Reports must be submitted within } 14 \text { days, } \\
\text { (within } 7 \text { days if making a claim for payment). }\end{array}$ & 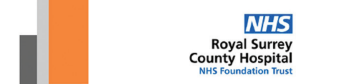 \\
\hline
\end{tabular}


process and address several of the highlighted issues. The leaflet provided clear instructions on how to access the ER system. It included examples of the various types of issues that can be reported and clarified which consultants should be receiving reports for on-call shifts. The leaflet was disseminated to all doctors (including consultants) within the trust via email. A teaching session was also held where the leaflet and the results of the questionnaires were presented to foundation trainees, and the impact it has on patient safety clearly highlighted.

Plan, do, study, act cycle 2

Following the introduction of the leaflet, the foundation trainees were re-surveyed 2 months later (supplemental material S2). The second questionnaire also featured questions regarding encouragement of the system by consultants and feedback on the guidance leaflet. The results of both questionnaires were compared and analysed using hypothesis testing and Z-score statistical testing to demonstrate significance.

To address the perceived lack of encouragement from consultants to ER, the results were presented at the consultant staff committee meeting along with a question and answer session.

\section{Results}

\section{Initial Questionnaire}

Thirty-six responses were received. Trainees collaboratively had worked overtime on 548 occasions; $20.3 \%$ of these episodes were documented in exception reports. The most common barriers for ER identified were having to have a meeting with the consultant to approve each report, fear of appearing inefficient and lack of encouragement by consultants. Other reasons included insufficient understanding of the submission process, uncertainty of the acceptable amount of overtime to report and lengthy delays in payment of reports submitted (Fig 2).

\section{Re-survey post-introduction of leaflet}

Thirty-one responses were received. Trainees collaboratively had worked overtime on 295 occasions. We showed a significant improvement in proportion of overtime exception reports filed from $20.3 \%$ to $33.9 \%$ ( $p<0.05$; Fig 3 ). There was no improvement in satisfaction with the ER process which was scored at a mean value of 3 out of 5 on both initial and re-survey. However, the majority $(80 \%)$ of respondents stated that they had found the leaflet useful. Almost half ( $48.3 \%$ ) of the foundation trainees reported that their consultant had not encouraged ER. At the consultant staff committee meeting, where all the above was

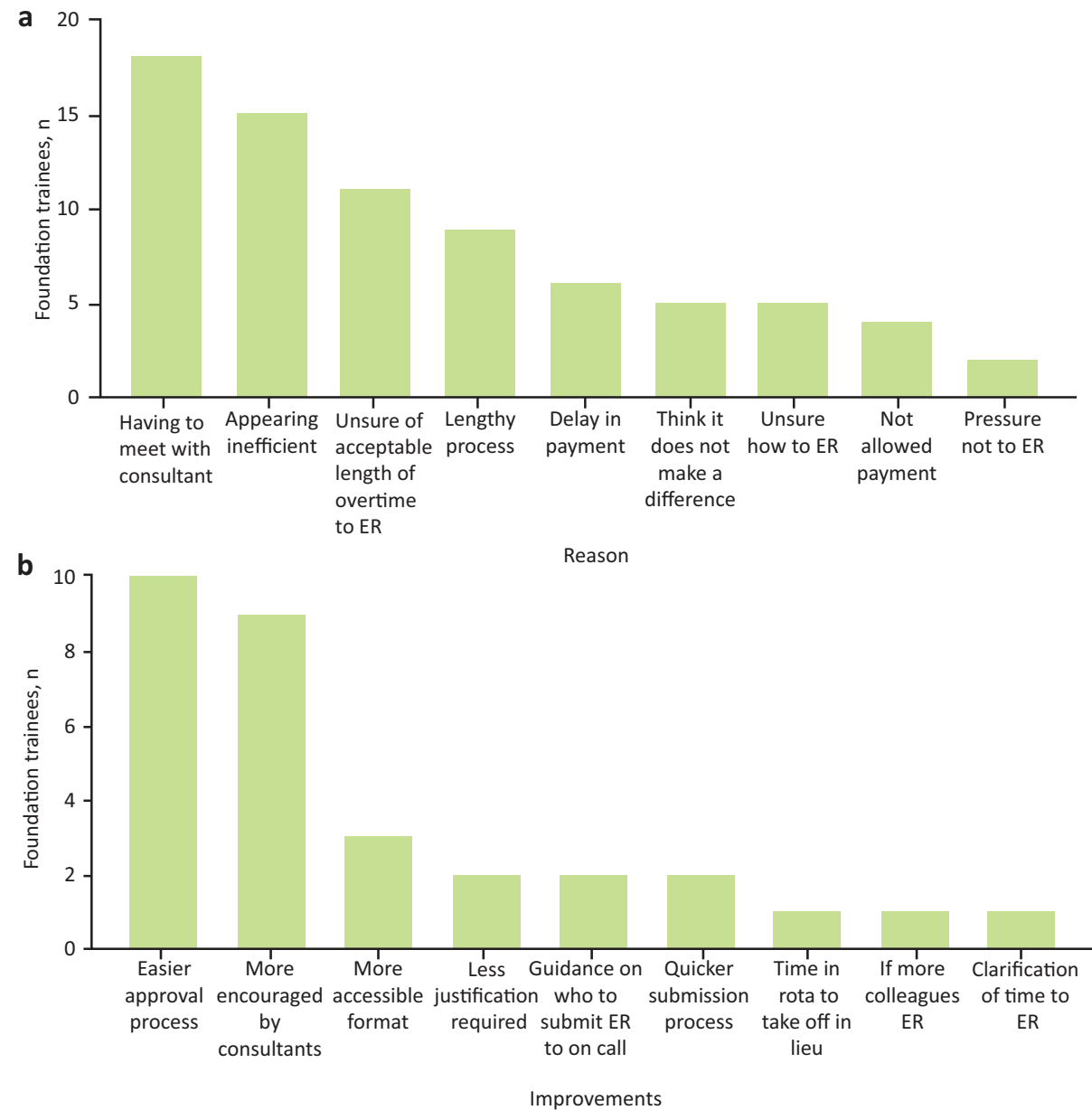

Fig 2. Results from initial questionnaire. a) Reasons given for not submitting exception reports. b) Improvements suggested to improve rates of exception reporting. ER = exception reporting. 


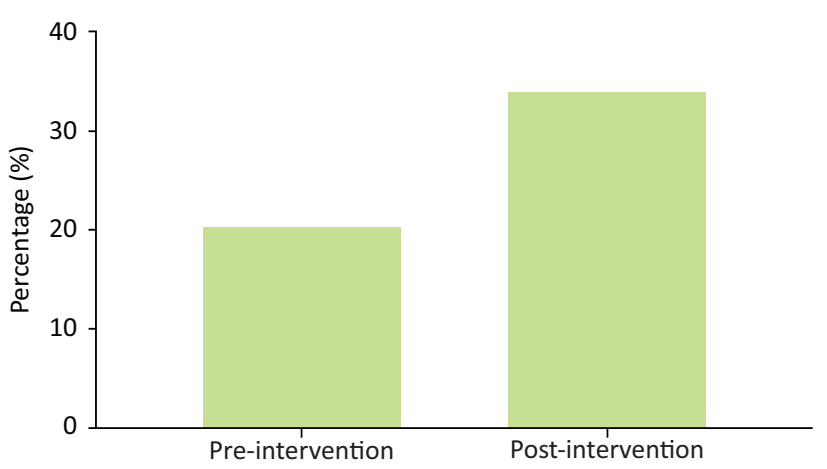

Fig 3. Proportion of overtime exception reported over a 2-month period pre- and post-intervention.

discussed, many consultants were in agreement about the importance of ER and were keen to improve the culture.

\section{Discussion}

ER is imperative in ensuring safe working hours for junior doctors. This helps to lead to changes that reduce fatigue and burnout and consequently improve patient safety. ${ }^{8,9}$ Although a relatively small sample size to analyse, we have demonstrated that there are several challenges with the ER system, such as a lack of awareness and understanding of the process.

The leaflet we created, and subsequent teaching session, sought to clarify the ER process and criteria, as well as highlight the importance of ER to make organisational changes. It aimed to improve the culture surrounding ER by explicitly stating that ER does not signify inefficiency, but rather is an important way of identifying high workload and lack of staffing. This addressed the barriers identified of insufficient understanding of the ER process, believing ER to have negligible impact and fear of appearing inefficient.

There were several challenges during this project. A few foundation trainees stated that they had not seen the newly designed leaflet. Several factors may have led to this. For example, the leaflet was distributed and presented at only one foundation teaching session. Therefore, trainees who could not attend, due to absence or high workload, would have missed the presentation. Efficiency improves with time. It is likely, during the period of this project from November till April, that foundation trainees had become much more efficient at their jobs thereby reducing the overall numbers of hours worked overtime. This may have contributed to the improvement in proportion of overtime exception reported.

We noted an increased uptake of exception reports submitted in the time during rotational changeover. This reflects foundation trainees starting a new specialty, and therefore working more overtime as they get used to a new system and structure of working.

Our study identified that $48 \%$ of foundation trainees were not encouraged to exception report. Kirwan et al showed at Barts Healthcare Trust that the uptake in ER was 35\%, with 29\% not encouraged to submit a report by their seniors. ${ }^{8}$ Another study has established that discouragement from seniors was a major contributor to low compliance with ER. ${ }^{6}$ As we also distributed leaflets to consultants, we noted more consultants encouraged their juniors to submit reports, addressing one of the most common barriers identified in the questionnaires.

Our project aimed to increase awareness and understanding of the ER process and a significant improvement in compliance with the system was seen. This may represent a consequence of our interventions and reflect the change we have initiated in the culture surrounding ER. Had there been wider circulation of the leaflet, there may have been a greater increase in ER. This project led to positive departmental changes, including the introduction of more locum doctors, and re-arrangement of the rota to enable more staff in the busier wards (such as respiratory and cardiology).

There are still, however, many challenges to be addressed.

Foundation trainee satisfaction rates with the ER process remained the same. This may be due to the processes that could not be influenced by the leaflet, for example, delays in payment and having to meet with the trainee's consultant to approve each report.

\section{Next steps}

As the majority of foundation trainees found the ER leaflet useful, it will be incorporated as part of the induction pack that juniors receive when they join the trust, with plans to extend this to core trainees and registrars.

We are in the process of creating a desktop link on all trust computers to the ER webpage. We also plan to liaise with Allocate (the ER software provider) regarding creation of an ER app to improve ease of access.

This study identified and addressed multiple factors contributing to the low compliance with the ER system. In order to safeguard staff wellbeing and thereby improve patient safety, we recommend that similar methods are employed in other NHS trusts.

\section{Supplementary material}

Additional supplementary material may be found in the online version of this article at www.rcpjournals.org/fhj:

S1 - Initial questionnaire to foundation trainees.

S2 - Second questionnaire to foundation trainees.

\section{References}

1 Collins A. Exclusive: Tens of thousands of junior doctors working beyond contract. HSJ 2019. www.hsj.co.uk/workforce/exclusivetens-of-thousands-of-junior-doctors-working-beyond-contract/ 7023993.article [Accessed 10 May 2019].

2 NHS Employers. Terms and conditions of service for NHS doctors and dentists in training (England): 2016. NHS, 2019.

3 British Medical Association. Rebranding for junior doctors. BMA, 2019. www.bma.org.uk/pay-and-contracts/pay/pay-banding/ rebanding-for-junior-doctors [Accessed 14 June 2020].

4 Hassan D, Maggs F. Barriers to the implementation of exception reporting at a busy district general hospital. FHJ 2019;6(Suppl 2):28

5 Cookson S. Rates of exception reporting at Royal Surrey County Hospital. Unpublished data, 2018.

6 NHS Improvement, British Medical Association, The Academy of Medical Royal College et al. Exception reporting: joint statement. NHS, 2018

7 West M, Coia D. Caring for doctors: Caring for patients. General Medical Council, 2019. www.gmc-uk.org/-/media/documents/ 
caring-for-doctors-caring-for-patients_pdf-80706341.pdf [Accessed 16 June 2020].

8 Kirwan C, Ali A, McCarten N. The junior doctor contract 2 years on: one trust's experience of exception reporting. $\mathrm{Br}$ ] Hosp Med (Lond) 2018;79:640-1.

9 Weigl M, Hornung S, Angerer P, Siergrist J, Glaser J. The effects of improving hospital physicians working conditions on patient care: a prospective, controlled intervention study. BMC Health Serv Res 2013;13:401.

Address for correspondence: Dr Dhanuja Senn, Royal Surrey County Hospital, Egerton Road, Guildford GU2 7XX, UK. Email: dhanuja.senn@nhs.net 